\title{
THE GEOMETRIC STRUCTURE OF SKEW LATTICES
}

\author{
JONATHAN LEECH
}

\begin{abstract}
A skew lattice is a noncommutative associative analogue of a lattice and as such may be viewed both as an algebraic object and as a geometric object. Whereas recent papers on skew lattices primarily treated algebraic aspects of skew lattices, this article investigates their intrinsic geometry. This geometry is obtained by considering how the coset geometries of the maximal primitive subalgebras combine to form a global geometry on the skew lattice. While this geometry is derived from the algebraic operations, it can be given a description that is independent of these operations, but which in turn induces them. Various aspects of this geometry are investigated including: its general properties; algebraic and numerical consequences of these properties; connectedness; the geometry of skew lattices in rings; connections between primitive skew lattices and completely simple semigroups; and finally, this geometry is used to help classify symmetric skew lattices on two generators.
\end{abstract}

Recall that a band is a semigroup satisfying the idempotent law: $x x=x$. Upon examining bands which are multiplicative subsemigroups of rings, one uncovers classes of bands which also possess an idempotent countermultiplication. This leads one to define a skew lattice to be an algebra with a pair of associative idempotent binary operations, the join and the meet, which are connected by a set of absorption laws (see 1.1). While skew lattices of idempotents in rings remain important sources of motivation, the results of [10-12] make it clear that skew lattices can sustain mathematical life on their own. Perhaps the most natural way to think about a skew lattice is as a noncommutative analogue of a lattice. As such, a skew lattice is not only an algebraic object, but also a geometric object. Thus far most of the research given in [10-12] has emphasized the algebraic side of skew lattices. The purpose of this paper is to investigate their geometric aspects and in particular the role of the natural partial order in determining their algebraic structure, much as a lattice is determined by its natural partial ordering. It is not our goal, however, to reinvent lattice theory. Hence the geometry of a skew lattice will be studied relative to the fixed structure of its underlying lattice. Saying this entails an implicit reference to the fundamental Clifford-McLean Theorem which in effect provides a first sketch of a skew lattice: a congruence is defined on each skew lattice (called natural equivalence) which induces its maximal lattice image and whose equiv-

Received by the editors November 20, 1989 and, in revised form, November 21, 1990.

1980 Mathematics Subject Classification (1985 Revision). Primary 20M10; Secondary 06A10, $20 \mathrm{M} 25$

Key words and phrases. Band, (partial) skew lattice, primitive skew lattice, partial ordering, quasi-ordering, coset, connected subalgebra. 
alence class are the maximal rectangular subalgebras of the skew lattice. (For details see 1.1 below and $[10,1.7]$.)

Our approach to skew lattice geometry is based on primitive skew lattices which, by definition, have precisely two maximal rectangular subalgebras. In the first section primitive skew lattices are studied by means of a natural decomposition of each equivalence class into cosets. There exists between each coset of the upper class and each coset of the lower class a coset bijection which is induced by the natural partial ordering. This coset decomposition together with the family of coset bijections provides the intrinsic geometry of the primitive skew lattice, which in turn determines its algebraic behavior (see 1.5, 1.6). Upon introducing coordinates one discovers that the data describing this coset geometry generalizes the data used in the Rees matrix description of completely simple semigroups. This leads to a connection between a subclass of primitive skew lattices and the class of completely simple semigroups. Examples of primitive skew lattices encountered in rings are all of this type.

An arbitrary skew lattice, provided it is not rectangular, may be viewed as being covered by its maximal primitive subalgebras. This leads one to consider how the various primitive geometries collectively form a global coset geometry on the entire skew lattice, which may in turn yield its algebraic structure. To do so, however, requires some preliminary developments which are of independent interest. Thus the second section begins by defining the natural graph of a skew lattice. It turns out that the connected components of a skew lattice form a congruence partition. Thus one obtains in Theorem 2.6 a complement to the above Clifford-McLean Theorem: the maximal connected subalgebras of a skew lattice form a congruence partition which induces the maximal rectangular image of the skew lattice. Next, the properties of being piecewise [dis-]connected and being homogeneous are defined and briefly discussed. The section concludes by "checking out" concepts and results encountered in the first two sections on classes of examples from rings.

The main application of connectedness occurs in the third section where it is used in 3.1 to define orthogonality of a given pair of equivalence classes in a mutually comparable third class. Theorem 3.3 asserts that any two equivalence classes are orthogonal in both their join class and their meet class; moreover individual joins and meets of pairs of elements from each class are determined by this orthogonality relationship. The algebraic structure of an arbitrary skew lattice is thus determined by its maximal primitive subalgebras and a global coset geometry is made possible. This geometry has numerous algebraic and numerical consequences which are explored in 3.4-3.6. These consequences are completely unlike anything occurring in either lattice theory or the theory of bands, and should serve to emphasize the profound structural differences existing between skew lattices and either bands or lattices. Next, partial skew lattices on quasi-ordered sets are introduced. Here the join and meet are defined only for elements comparable under the given quasi-order. The point of view which we adopt is that partial skew lattices are natural mathematical objects. Indeed they may be viewed as noncommutative partially ordered sets. (The full set of idempotents in any ring forms a partial skew lattice in at least three different ways, two of which are mentioned in 3.8(ii).) Thus an obvious question is raised: what are the precise conditions under which a partial skew lattice defined on a quasi-ordered set naturally induces a full skew lattice structure on the set? 
This question receives a simple answer in Theorem 3.10. This is, perhaps, the result coming closest to the structure theorems for bands given by Petrich in [15] and Warne in [19], although it is clearly more geometric in character than the theorems given by the other authors. Partial skew lattices may in turn be viewed as quasi-ordered sets which are covered by primitive skew lattices. Thus to complete the picture, attention is focused on determining when such a primitive covering induces a partial skew lattice structure on the quasi-ordered set. The answer, given in Theorem 3.12 in terms of coset projections, directly generalizes the picture of normal bands given by Yamada and Kimura in [20]. The section concludes with a discussion of partial skew lattices for which coset bijections between comparable equivalence classes form a category under the standard composition of partial bijections. Theorem 3.16 contains several pertinent facts about such categorical partial skew lattices.

The final section consists of further examples and results which apply and illustrate the theory of the first three sections. Skew chains in rings are studied in 4.1-4.5. Next, several classes of skew lattices are briefly discussed in 4.6-4.8. The paper concludes by classifying symmetric skew lattices on two generators in $4.10-4.12$.

Detailed background on skew lattices may be found in [10]. Both [8 and 17] are good sources of information on bands. Information on completely simple semigroups may be found in $[2,8,13]$ or [16].

\section{COSETS AND COORDinAtes}

1.1. A skew lattice is an algebra $(S, \vee, \wedge)$ where both $\vee$ and $\wedge$ are associative, idempotent binary operations which jointly satisfy the absorption identities:

$$
x \wedge(x \vee y)=x=x \vee(x \wedge y) \text { and }(x \vee y) \wedge y=y=(x \wedge y) \vee y
$$

Given that the operations are associative and idempotent, these identities are equivalent to the following dualities: $x \vee y=x$ iff $x \wedge y=y$ and $x \vee y=y$ iff $x \wedge y=x$. Every skew lattice has a natural partial ordering defined by $x \geq y$ iff $x \wedge y=y=y \wedge x$, or dually, $x \vee y=x=y \vee x$. Given nonempty sets, $L$ and $R$, the product $L \times R$ becomes a skew lattice upon defining $(x, y) \vee$ $\left(x^{\prime}, y^{\prime}\right)=\left(x^{\prime}, y\right)$ and $(x, y) \wedge\left(x^{\prime}, y^{\prime}\right)=\left(x, y^{\prime}\right)$. Any isomorphic copy of such a skew lattice is said to be rectangular. Recall from $[10,1.7]$ that the maximal rectangular subalgebras of a skew lattice form a congruence partition, with the induced quotient algebra being the maximal lattice image of the given skew lattice. The actual congruence, called natural equivalence, is given by $x \equiv y$ if both $x \vee y \vee x=x$ and $y \vee x \vee y=y$ or dually, $x \wedge y \wedge x=x$ and $y \wedge x \wedge y=y$.

1.2. A primitive skew lattice is a skew lattice with precisely two equivalence classes. If $A$ and $B$ denote the two classes, then $A>B$ means that $A$ is the higher class. In this case, by a coset of $A$ in $B$ is meant any subset of form $A \wedge b \wedge A$ for $b$ in $B$, and for any $a$ in $A$ its image in $B$ is the subset $a \wedge B \wedge a=\{b$ in $B \mid b \leq a\}$. Cosets and image sets in $A$ are defined in dual fashion. To explain their role, it is helpful to recall that skew lattices are biregular, that is, both $\vee$ and $\wedge$ satisfy the identity: $x u v x=x u x v x$. (See $[10,1.15]$.) A useful alternative to this identity is the regularity implication: if 
$x, y, z$ are equivalent, then $x u v z=x u y v z$. We now present the following basic lemma.

Lemma 1.3. Let $S$ be a primitive skew lattice with equivalence classes $A>B$. Then

(i) $B$ is partitioned by the cosets of $A$. In detail, $b$ is in $A \wedge b \wedge A$ for all $b$ in $B$, and if $x$ is in $A \wedge b \wedge A$, then $A \wedge x \wedge A=A \wedge b \wedge A$.

(ii) The image set in $B$ of any $a$ in $A$ is a transversal of cosets of $A$ in $B$. Dual remarks hold for cosets and image sets of $B$ in $A$. Furthermore:

(iii) Given cosets, $B \vee a \vee B$ in $A$ and $A \wedge b \wedge A$ in $B$, there is a natural bijection of cosets with $x$ in $B \vee a \vee B$ corresponding to $y$ in $A \wedge b \wedge A$ iff $x \geq y$.

(iv) The meet and join operations are determined by the coset bijections.

Proof. By absorption $b=(a \vee b) \wedge b \wedge(b \vee a)$ so that $b$ is in $A \wedge b \wedge A$. Given $x$ in $A \wedge b \wedge A$, say $x=m \wedge b \wedge n$ for $m, n$ in $A$, then regularity implies that for all $a, a^{\prime}$ in $A, a \wedge x \wedge a^{\prime}=a \wedge b \wedge a^{\prime}$. Thus (i) is seen. We next verify (iii). Let $a$ in $A$ and $b$ in $B$ be given. Then for each $x$ in $B \vee a \vee B, x \geq x \wedge b \wedge x$ and we just saw that $x \wedge b \wedge x=x \wedge y \wedge x$ for each $y$ in $A \wedge b \wedge A$. Thus $\geq$ induces a function between cosets and by the dual argument this function is in fact a bijection. (ii) follows immediately from (iii). To see (iv), let $a$ in $A$ and $b$ in $B$ be given. Then $a \wedge b=a^{\prime} \wedge b$ in $B$ where $a^{\prime}=a \wedge b \wedge a$ is the unique image of $a$ in the coset of $A$ to which $b$ belongs. Likewise $b \wedge a=b \wedge a^{\prime}$. The cases $a \vee b$ and $b \vee a$ are handled in dual fashion.

1.4. The above lemma leads to a general construction. But first recall that a skew lattice is right handed if it satisfies the identity $x \wedge y \wedge x=y \wedge x$ and hence also its dual $x \vee y \vee x=x \vee y$. These identities essentially assert that in each equivalence class $x \wedge y=y$ and $x \vee y=x$. Left handed skew lattices are defined by the opposite identities. In what follows a right [left] handed primitive skew lattice is simply called right [left] primitive. Due to biregularity, every primitive skew lattice factors as the fibered product of a right primitive skew lattice with a left primitive skew lattice over the two element lattice, with both factors being unique to within isomorphism. (See [10, 1.14-1.16].) Both left and right primitive skew lattices may be constructed from the following class of neutral objects.

1.5. A $P$-graph consists of a pair of partitioned sets, $A=\bigcup A_{i}$ and $B=$ $\bigcup B_{j}$, for which the cells from both partitions have a common cardinality, together with a fixed set of cell bijections $\phi_{j i}: A_{i} \rightarrow B_{j} . P$-graphs are denoted by pairs of the form $\left(A_{i}, B_{j}\right)$. For small orders, $P$-graphs are easily drawn.

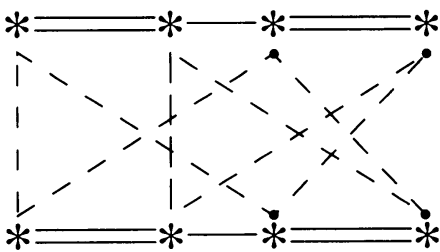


In this diagram the cells are indicated by the double bonding and the bijections are indicated by the broken lines. Given a $P$-graph $\left(A_{i}, B_{j}\right)$ with $A=\bigcup A_{i}$ and $B=\bigcup B_{j}$, we induce a right primitive structure on $A \cup B$ as follows. The operations restricted to $A$ or $B$ are trivial: $x \wedge y=y$ and $x \vee y=x$. Given $x$ in $A_{i}$ and $y$ in $B_{j}$, then again $x \wedge y=y$ and $x \vee y=x$; however, $y \wedge x=\phi_{j i}(x)$ and $y \vee x=\phi_{j i}^{-1}(y)$. A right primitive skew lattice $P\left[A_{i}, B_{j}\right]$ is thus obtained with equivalence classes $A>B$, with cosets being the $A_{i}$ and the $B_{j}$, and with the coset bijections being the $\phi_{j i}$. Upon dualizing the definitions of $\vee$ and $\wedge$ one obtains the left primitive dual, $P^{\prime}\left[A_{i}, B_{j}\right]$. By Lemma 1.3, it follows that to within isomorphism, every right [left] primitive skew lattice is obtained in this fashion. If 2 denotes the lattice $1^{0}$, then the discussion of this section up till now may be summarized as follows:

Theorem 1.6. If $P$ is a primitive skew lattice, then it has a fibered product decomposition, $P \cong\left[A_{i}, B_{j}\right] \times_{2} P^{\prime}\left[C_{k}, D_{l}\right]$, where $\left(A_{i}, B_{j}\right)$ and $\left(C_{k}, D_{l}\right)$ denote $P$-graphs. Both P-graphs are unique to within isomorphism of $P$-graphs.

1.7. We introduce coordinates. Let $I$ and $J$ be nonempty sets, let $u$ : $G \times C \rightarrow C$ be an action of group $G$ on set $C$, and let $\theta: J \times I \rightarrow G$ be a map. Partition $A=I \times C$ into cells $\{i\} \times C$, partition $B=J \times C$ into cells $\{j\} \times C$, and given $j, i$ define $\phi_{j i}:\{i\} \times C \rightarrow\{j\} \times C$ by $\phi_{j i}(i, c)=(j, \theta(j, i) c)$. We thus obtain a $P$-graph.

The right primitive skew lattice constructed from this $P$-graph is denoted by $P[I, J, u, \theta]$. Both the $P$-graph and the skew lattice are normalized at $(i, j)$ if $\theta[\{j\} \times I \cup J \times\{i\}]=1$ in $G$. If $P$ is a given right primitive skew lattice, then an isomorphism $P \cong P[I, J, u, \theta]$ is called a coordinatization of $P$. The left handed dual to $P[I, J, u, \theta]$ is denoted $P^{\prime}[I, J, u, \theta]$.

Theorem 1.8. Any right [left] primitive skew lattice can be given a normalized coordinatization.

Proof. Let $P$ be a right primitive with classes $A>B$, with coset decompositions $A=\bigcup A_{i}$ and $B=\bigcup B_{j}$, and with coset bijections $\phi_{j i}: A_{i} \rightarrow B_{j}$. Pick a coset from each class, say $A_{0}$ and $B_{0}$. For each pair $i, j$ define the permutation $\theta(j, i)$ on $A_{0}$ by $\theta(j, i)=\phi_{j 0}^{-1} \phi_{j i} \phi_{0 i}^{-1} \phi_{00}$. Let $G$ be any permutation group on $A_{0}$ containing all the $\theta(j, i)$ and let $u: G \times A_{0} \rightarrow A_{0}$ be the natural action. If $I$ and $J$ are indexing sets for the $A_{i}$ and $B_{j}$ respectively, then define the bijection $\sigma: P \rightarrow P[I, J, u, \theta]$ on cosets by $\sigma \mid A_{i}=\{i\} \times \phi_{00}^{-1} \phi_{0 i}$ and $\sigma \mid B_{j}=\{j\} \times \phi_{j 0}^{-1}$. These restrictions of $\sigma$ commute with the coset bijections so that $\sigma$ is an isomorphism of skew lattices. Finally, the constructed skew lattice is clearly normalized at $(0,0)$.

1.9. A special case of coordinatization occurs when $G=C$ and $u: G \times G \rightarrow$ $G$ is group multiplication. In this case the data reduces to the indexing sets, $I$ and $J$, the group $G$ and a map $\theta: J \times I \rightarrow G$. The resulting skew lattice is denoted by $P[I, J, G, \theta]$, or in the left-handed case by $P^{\prime}[I, J, G, \theta]$. If $P$ is a primitive skew lattice, then an isomorphism of $P$ with either $P[I, J, G, \theta]$ or its dual is said to be a coordinatization with group translations. Such a coordinatization can always be chosen to be normalized. It is well known that the data $[I, J, G, \theta]$ is precisely what is needed to carry out the general construction of a completely simple semigroup: with the given data define a multiplication on 
$I \times G \times J$ by $(i, a, j)\left(i^{\prime}, b, j^{\prime}\right)=\left(i, a \theta\left(j, i^{\prime}\right) b, j^{\prime}\right)$. The completely simple semigroup thus constructed is denoted by $S[I, J, G, \theta]$. This "coincidence" suggests that primitive skew lattices which are coordinatized with group translations can be constructed from completely simple semigroups. To see this let $S$ be a completely simple semigroup and pick an idempotent $e$ in $S$. Both $S e$ and $e S$ decompose into maximal subgroups with the group $e S e$ being the intersection. If we ignore the minor inconvenience of nonempty intersection (which is easily repaired), then $S e \cup e S$ can be turned into a right primitive skew lattice with classes $S e>e S$ and with cosets being the maximal subgroups. One need only indicate the coset bijections: for idempotents $f$ in $S e$ and $g$ in $e S$, then the coset bijection between the corresponding subgroups, $\phi: f S f \rightarrow g S g$, is given by $\phi(s)=g s g$. If $S=S[I, J, G, \theta]$ with $\theta$ normalized at $i=0$ and $j=0$, then upon choosing $e=(0,1,0)$ the right primitive skew lattice constructed in this fashion is a copy $P[I, J, G, \theta]$. For upon picking $s=(i, a, 0)$ in $f S f$, where $f=(i, 1,0)$, and idempotent $g=(0,1, j)$ we calculate $g s g=(0, \theta(i, j) a, j)$ which is essentially the rule for $\phi:\{i\} \times G \rightarrow\{j\} \times G$. For example, let $I=J=G=Z_{2}$, the field of order two, where the group structure is the field addition and $\theta$ is just field multiplication. Then the $P$-graph of $P\left[Z_{2}, Z_{2}, Z_{2}, \theta\right]$ is given in the diagram of 1.5. This graph is deformed in the semigroup $S\left[Z_{2}, Z_{2}, Z_{2}, \theta\right]$.

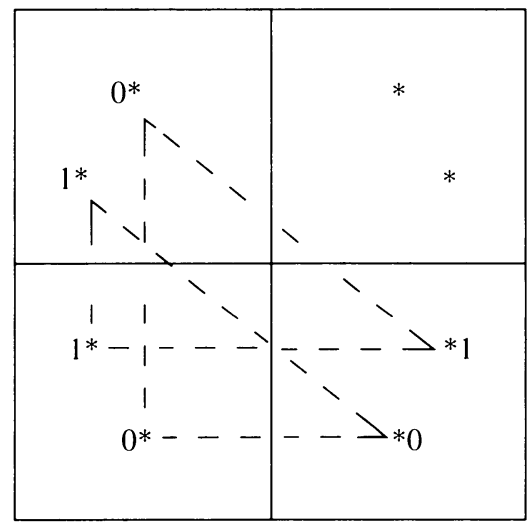

Here the group in the lower left corner does double duty as a coset in both classes.

\section{ConNectedness}

2.1. The natural graph of a skew lattice $S$ is the undirected graph $(S, E)$ given by the natural partial ordering of $S$. Thus the vertices are the elements of $S$ while $\{x, y\}$ forms an edge of the graph when either $x>y$ or $y>x$. The skew lattice is connected if its graph is connected. In general, a component of $S$ is any connected component of its graph. Clearly each component has nonempty intersection with each equivalence class of $S$. Moreover, for the case when $S$ is the primitive skew lattice $P$, then each component of $P$ has nonempty intersection with each coset. This leads us to say that a primitive skew lattice $P$ is disconnected if in any, and hence every, coset of $P$ distinct elements of the coset belong to distinct components. $P$ is degenerate if it is 
both connected and disconnected, in which case $x>y$ holds between all $x$ in the higher class and all $y$ in the lower class. By a coset component of $P$ is meant the intersection of a coset of $P$ with a component of $P$. The situation for primitive skew lattices is summed up in the following theorem whose rather easy proof is left to the reader.

Theorem 2.2. Let $P$ be a primitive skew lattice. Then the components of $P$ are the maximal connected subalgebras of $P$ and the component partition of $P$ is a congruence partition for which the induced quotient algebra is the maximal rectangular image of $P$. Moreover the coset components of $P$ form a congruence partition for which the induced quotient algebra is the maximal disconnected image of $P$. Finally, if $P$ is disconnected, then it factors as the product of a degenerate skew lattice with a rectangular skew lattice.

2.3. Let $P$ be right primitive with normal coordinatization $P[I, J, u, \theta]$. Then $P$ is connected when $K$, the subgroup generated by $\theta[J \times I]$, acts transitively on its set. In general the components of $P$ correspond to the orbits of $K$. Thus $P$ is disconnected when $\theta[J \times I]=1$. Suppose that $P$ is normally coordinatized with group translations, say by $P[I, J, G, \theta]$. In this case the components of $P$ correspond to the subgroup cosets of $K$ in $G$, so that connectedness occurs when $\theta[J \times I]$ generates $G$. Upon passing to the associated completely simple semigroup, connectedness corresponds to the idempotents generating the semigroup, while disconnectedness corresponds to the idempotents forming a subsemigroup.

In this paper, a connected graph with each vertex having degree two is called a simple circuit when finite, and an infinite simple path when infinite.

Theorem 2.4. Let $P$ be a right primitive skew lattice. Then the natural graph of $P$ is a simple circuit precisely when $P$ has a coordinatization with group translations of the form $P\left[Z_{2}, Z_{2}, Z_{n}, \theta\right]$ for some $n \geq 1$ where $\theta(j, i)=j i$ for $i, j$ in $\{0,1\}$. The graph is an infinite simple path precisely when $P$ has a coordinatization $P\left[Z_{2}, Z_{2}, Z, \theta\right]$ where again $\theta(j, i)=j i$, in which case $P$ is an infinite primitive skew lattice on four generators. (All infinite primitive skew lattices need at least four generators.)

2.5. Returning to the general case of an arbitrary skew lattice $S$, the skew lattice is said to be bounded if it has both a maximal and a minimal equivalence class, in which case both classes combine to form the boundary algebra of $S$, denoted $\operatorname{Bd}(S)$. More generally, if $B>C$ is a comparable pair of equivalence classes in $S$, then the interval $[C, B]$ is the subalgebra obtained from the union of $B$ and $C$ with all intermediate equivalence classes. We now present the complement to the Clifford-McLean Theorem.

Theorem 2.6. The components of a skew lattice $S$ are its maximal connected subalgebras. Moreover, the partition of $S$ into components is a congruence partition for which the induced quotient algebra is the maximal rectangular image of $S$. If $S$ is also bounded with boundary algebra $\operatorname{Bd}(S)$, then the inclusion $\operatorname{Bd}(S) \subseteq S$ induces an isomorphism of maximal rectangular images.

Proof. The theorem holds when $S$ is primitive. So assume $S$ is bounded with maximal class $A$ and minimal class $Z$. Then $\operatorname{Bd}(S)=A \cup Z$ decomposes into its components $A_{i} \cup Z_{i}$. An element $x$ in $S$ is said to belong to component 
$A_{i} \cup Z_{i}$ if $A_{i} \cup Z_{i}$ is the unique boundary component such that there exist $u$ in $A_{i}$ and $v$ in $Z_{i}$ such that $u \geq x \geq v$. For any $y$ in $S$ such that either $x \geq y$ or $y \geq x$ it is clear that $y$ belongs to the same boundary component as $x$. Hence the inclusion $\operatorname{Bd}(S) \subseteq S$ does induce a bijection of components. Let $x$ belong to $A_{i} \cup Z_{i}$ and $y$ belong to $A_{j} \cup Z_{j}$. Pick $u$ in $A_{i}$ and $w$ in $A_{j}$ so that $u \geq x \vee y \vee x$ and $w \geq y \vee x \vee y$. Thus $u \vee w \geq(x \vee y \vee x) \vee(y \vee x \vee y)=x \vee y$ and $x \vee y$ must lie in the component of $S$ containing $\left(A_{i} \cup Z_{i}\right) \vee\left(A_{j} \cup Z_{j}\right)$. Likewise $x \wedge y$ lies in the component containing $\left(A_{i} \cup Z_{i}\right) \wedge\left(A_{j} \cup Z_{j}\right)$. The bounded case of the theorem now follows from the primitive case. In general, any two elements connected in a skew lattice are also connected in some interval. The general case of the theorem follows from the fact that every skew lattice is the directed union of its intervals.

2.7. A skew lattice is piecewise connected [disconnected] if each of its maximal primitive subalgebras is [dis-]connected. Clearly piecewise connected implies connected; however, a skew lattice can be simultaneously connected and piecewise disconnected with all its maximal primitive subalgebras having infinitely many components. For let $X$ denote the set of infinite 0-1 binary sequences which are eventually 0 , and for each integer $n \geq 0$ let $X_{n}$ be a copy of $X$. Let $S$ be the right-handed skew lattice with classes $X_{0}>X_{1}>\cdots$ determined by letting each primitive subalgebra $X_{m}>X_{n}$ have unique lower coset $X_{n}$ with the upper cosets in $X_{m}$ consisting of sequences sharing a common prefix of length $n-m$ and with the coset bijections being maps deleting these prefixes. Since all sequences eventually vanish $S$ is connected. Since each lower class is infinite, each maximal primitive subalgebra is disconnected with infinitely many components. On the other hand, any connected skew lattice having a maximal primitive subalgebra with finitely many components must also have a connected maximal primitive subalgebra.

2.8. A skew lattice is homogeneous if it factors as the product of a rectangular algebra with a connected algebra. Any primitive skew lattice which can be coordinatized with group translations is homogeneous. In particular, disconnected primitive skew lattices are homogeneous. A skew lattice $S$ is called binormal if both $x \vee S \vee x$ and $x \wedge S \wedge x$ are sublattices for all $x$ in $S$, in which case the components of $S$ are precisely the maximal sublattices of $S$. Schein proved that binormal skew lattices are homogeneous with lattice components. (See [18]; this also follows from the Clifford-McLean theorem and Theorem 2.6.) The maximal binormal image of a skew lattice is the quotient algebra induced from the intersection of the component congruence with natural equivalence. Binormal skew lattices form a subvariety of skew lattices.

2.9. Let $A$ be a ring with $E(A)$ denoting its set of idempotents. In this paper all rings have units. For any idempotent $e$ the $R$-set of $e$ is the set

$$
R(e)=e+e A(1-e)=\{x \mid e x=x, x e=e\} .
$$

The $R$-sets partition $E(A)$ into its maximal right rectangular bands. They are naturally ordered upon setting $R(e) \geq R(f)$ whenever $e f=f$, or equivalently $x y=y$ for all $x$ in $R(e)$ and $y$ in $R(f)$. If $R(e) \geq R(f)$ but $R(e) \neq R(f)$, then we write $R(e)>R(f)$ and call the pair a primitive pair of $R$-sets. Recall the binary operations defined on $A: x \vee y=x+y-x y$ and $x \wedge y=x y$. Using these operations, every primitive pair of $R$-sets forms a maximal right primitive skew lattice in $A$. Assuming $e>f$ for the displayed representatives, this skew 
lattice is denoted by $P[e, f] . P[e, f]$ has the coordinatization $P[I, J, C, \theta]$ described as follows: $I=(e-f) A(1-e), J=f A(e-f), C=f A(1-e)$ and $\theta: J \times I \rightarrow C$ is just restricted multiplication. Using the decompositions $e A(1-e)=I+C$ and $f A(1-f)=J+C$, the isomorphism of $P[e, f]$ with $P[I, J, C, \theta]$ sends $e+i+c$ to $(i, c)$ and $f+j+c$ to $(j, c)$. Each $P[e, f]$ is thus homogeneous; it is also connected when $J I=f A(e-f) A(1-e)$ generates $C=f A(1-e)$. Hence each $P[e, f]$ is connected when the ring is simple. The pieces of $P[e, f]$ are seen in the matrix diagram:

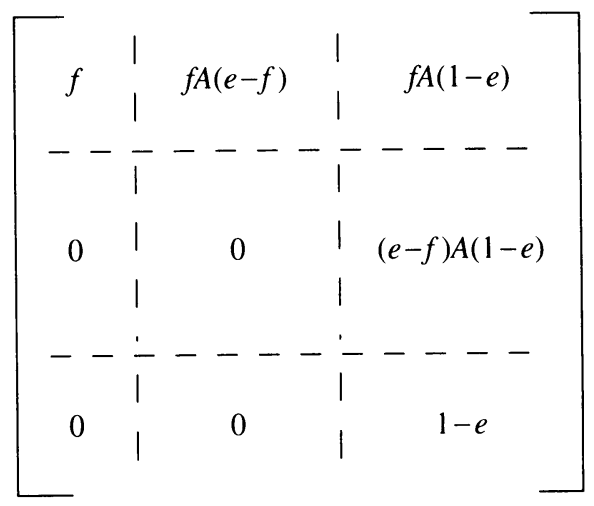

2.10. Adjacent to the skew lattice $P[e, f]$ lies a completely simple semigroup. If $g$ denotes $f+(1-e)$, then the companion semigroup $S=S[e, f]$ is the multiplicative subsemigroup of $A$ given by $S=g+I+C+J$ where $I, J$, and $C$ are as in the above paragraph. $S$ is easily seen to be completely simple. In particular, $S g=g+I+C$ has $g+I$ for its idempotents, $g S=g+C+J$ has $g+J$ for its idempotents, and the maximal subgroup at $g$ is $g S g=g+C$. Given $x=g+i+c$ in the subgroup of idempotent $g+i$ in $S g$ and given idempotent $h=g+j$ in $g S$, multiplication yields $h x h=h+c+j i$. Thus the right primitive skew lattice constructed on $S g \cup g S$ is just a copy of $P[e, f]$. As evidence of the naturalness of this correspondence, observe that if $d>e>f$ is a chain in $E(A)$, then under standard subset multiplication, $S[d, e] S[e, f]=S[d, f]$ in $A$. It follows from 3.16 below that in contravariant fashion $P[e, f] P[d, e]=P[d, f]$.

2.11. We pass to a significantly larger class of skew lattices inside a ring. A right handed skew lattice $S$ in $(A, \vee, \wedge)$ is affine if for every finite set of equivalent elements $x_{1}, \ldots, x_{n}$ in $S$, each affine combination $k_{1} x_{1}+\cdots+k_{n} x_{n}$ belongs to their common equivalence class, where $k_{1}+\cdots+k_{n}=1$ and the $k_{i}$ lie in $K$, the center of $A$. Given a right-handed skew lattice $S$ the affine closure of $S$ in $A$ is the set of all affine combinations of equivalent elements in $S$. The affine closure is easily seen to be the minimal affine skew lattice in $A$ containing $S$. For any $e>f$ in $E(A), P[e, f]$ is affine. It is not hard to show that the affine primitive subalgebras of $P[e, f]$ containing $e>f$ are precisely the subalgebras of the form $(e+M+C) \cup(f+N+C)$ where here $M, N$, and $C$ are $K$-submodules of $(e-f) A(1-e), f A(e-f)$ and $f A(1-e)$ respectively and for which $N M \subseteq C$. Most types of right-handed skew lattices in rings considered in [10 and 11] are affine. For such skew lattices we have the following results. 
Theorem 2.12. If $A$ is a ring and $S$ is an affine right-handed skew lattice in $(A, \vee, \wedge)$, then all the components of $S$ are affine. If $S$ is also bounded, then it is homogeneous.

Proof. First assume $S$ is bounded with boundary $\operatorname{Bd}(S)=(e+M+C) \cup$ $(f+N+C)$ as in the above paragraph. If $C^{\prime}$ is the submodule generated by $N M$, then the necessarily affine component of $\operatorname{Bd}(S)$ containing $e>f$ is $\left(e+M+C^{\prime}\right) \cup\left(f+N+C^{\prime}\right)$. Let $S^{\prime}$ denote the corresponding component of $S$ and let $x$ and $y$ lie in a common class of $S^{\prime}$. Then for any $v$ in $f+N+C^{\prime}$, both $v x$ and $v y$ lie in $f+N+C^{\prime}$ also. If $a+b=1$ in center $K$, then $a x+b y \geq v(a x+b y)=a v x+b v y$. Since $f+N+C^{\prime}$ is affine, $a v x+b v y$ lies in $f+N+C^{\prime}$ and hence $a x+b y$ lies in $S^{\prime}$ which is thus seen to be affine. Next note that for all $x$ in $S$ and $c, d$ in $C, c x=0, x d=d$, and $c d=0$. Thus for all $x, y$ in $S$ and $c, d$ in $C$ :

$$
(x+c) \wedge(y+d)=(x \wedge y)+d \text { and }(x+c) \vee(y+d)=(x \vee y)+c \text {. }
$$

Thus every translate $S^{\prime}+c$ for $c$ in $C$ is also a (necessarily affine) component of $S$; moreover, any two such translates are equal, $S^{\prime}+c=S^{\prime}+d$, precisely when $c-d$ lies in $C^{\prime}$. Let $T$ be a transversal of the $C^{\prime}$-cosets in $C$; then the bijection of $S$ with $S^{\prime} \times T$ sending each $x$ in $S$ to the unique pair $\left(x^{\prime}, c\right)$ in $S^{\prime} \times T$ for which $x=x^{\prime}+c$ is an isomorphism by the above identities. That all components of $S$ are affine also in the unbounded case follows again from $S$ being the directed union of its (necessarily affine) intervals.

Corollary 2.13. Let $A$ be a ring and let $S$ be a connected, right handed skew lattice in $(A, \vee, \wedge)$. Then the affine closure of $S$ is also connected.

\section{ORTHOGONALITY AND PARTIAL SKEW LATTICES}

3.1. Consider the general situation of three equivalence classes, $A, B$, and $C$, such that $C$ is comparable to both $A$ and $B$. By a class component of $A$ in $C$ is meant the intersection of a component of $A \cup C$ with $C$. One similarly defines a class component of $B$ in $C$. We say that $A$ and $B$ are orthogonal in $C$ if each class component of $A$ [of $B$ ] in $C$ is contained in a unique coset of $B$ [of $A$ ] in $C$. The orthogonality of $A$ and $B$ in $C$ is equivalent to asserting that each $x$ in $A$ is covered by a coset of $B$ in $C$, that is, the image set of $x$ in $C$ is a subset of this coset of $B$, and likewise each $y$ in $B$ covered by a coset of $A$ in $C$. If $A$ and $B$ are orthogonal in $C$, then each coset of $A$ in $C$ has nonempty intersection with each coset of $B$ in $C$; moreover, all such coset intersections have common cardinality. For let $A_{1}, A_{2}$ be cosets of $A$ in $C$; let $B_{1}, B_{2}$ be cosets of $B$ in $C$; and let $\phi_{1}, \phi_{2}$ denote coset bijections from $A_{1}$ and $A_{2}$ onto a common coset in $A$. The bijection $\phi_{2}^{-1} \phi_{1}: A_{1} \rightarrow A_{2}$ and its inverse $\phi_{1}^{-1} \phi_{2}$ keep individual elements in the same class component of $A$ in $C$. Orthogonality implies both bijections restrict to an inverse pair of bijections of $A_{1} \cap B_{1}$ with $A_{2} \cap B_{1}$. A similar argument shows that $A_{2} \cap B_{1}$ is in bijective correspondence with $A_{2} \cap B_{2}$, so that the assertion is verified. (We thus visualize $C$ to be a doubly partitioned rectangle arising from a horizontal partition by one class of congruent rectangles, the $A$-cosets, and a vertical partition by a second class of congruent rectangles, the $B$-cosets.)

3.2. What happens when $J$ is the join class of $A$ and $B$, and $M$ is their meet class? If $x$ in $A$ and $y$ in $B$ are given, then $y \wedge x \wedge y=y \wedge u \wedge y$ for 
any $u$ in $M$ such that $u \leq x$. Consequently, each $x$ in $A$ is covered by a fixed coset of $B$ in $M$; likewise each $y$ in $B$ is covered by a coset of $A$ in $M$. Thus $A$ and $B$ are orthogonal in their meet class $M$; dually, they are orthogonal in their join class $J$. Since $x \wedge y=(x \wedge v \wedge x) \wedge(y \wedge u \wedge y)$ for any $x$ in $A, y$ in $B$, and $u, v$ in $M$, where $u \leq x$ and $v \leq y$, it follows that $x \wedge y=x^{\prime} \wedge y^{\prime}$ where $x^{\prime} \leq x$ and $y^{\prime} \leq y$ in $M$ are the unique images in the cosets covering $y$ and $x$ respectively. To sum up:

Theorem 3.3. Let $A$ and $B$ be equivalence classes in a skew lattice. Then $A$ and $B$ are orthogonal in both their join class $J$ and their meet class $M$. For each $x$ in $A$ and $y$ in $B, x \wedge y=x^{\prime} \wedge y^{\prime}$, where $x^{\prime}$ in $M$ is the image of $x$ in the unique coset of $A$ covering $y$, and likewise $y^{\prime}$ in $M$ is the image of $y$ in the coset of $B$ covering $x$. The computation of $x \vee y$ is determined in dual fashion.

3.4. The double partition of either $J$ or $M$ by $A$-cosets and $B$-cosets is illustrated in the following diagram in which the partition is shown to be refined by the coset partition which $J$ and $M$ directly induce on each other.

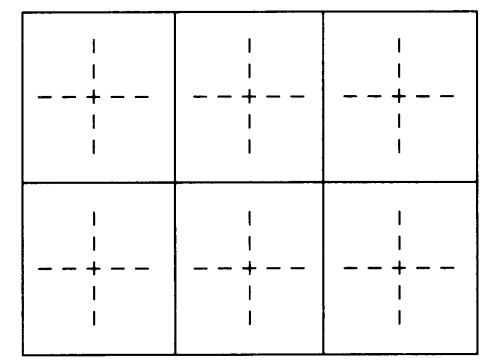

To see when the double partition coincides with the partition by $J-M$ cosets, recall that a skew lattice is symmetric if commutativity is unambiguous: $x \vee y=y \vee x$ iff $x \wedge y=y \wedge x$. Symmetric skew lattices form a subvariety of skew lattices. The examples in rings studied in [10 and 11] are all symmetric.

Theorem 3.5. A skew lattice is symmetric if and only if for any two equivalence classes, $A$ and $B$ : (i) the partition of the join class $J$ by intersections of $A$ cosets with $B$-cosets equals the partition by cosets of the meet class $M$; (ii) the dual assertion holds for the meet class $M$.

Proof. First assume that in $J$ and $M, A$-cosets and $B$-cosets intersect to $J-M$ cosets. Let $a$ in $A$ and $b$ in $B$ be given with $a \vee b=b \vee a$. By orthogonality, $a \wedge b$ and $b \wedge a$ lie in an $A-B$ coset intersection. Thus $a \wedge b$ and $b \wedge a$ lie in a common $J$-coset, and since $a \vee b \geq a \wedge b, b \wedge a$, it follows that $a \wedge b=b \wedge a$. Similarly $a \wedge b=b \wedge a$ implies $a \vee b=b \vee a$. Suppose instead that more than one $J$-coset in $M$ lies inside the intersection $I$ of an $A$-coset with a $B$-coset. Thus there exists $j$ in $J$ having at least two distinct images in $I$. Form a nonsymmetric subalgebra $J^{\prime} \cup A^{\prime} \cup B^{\prime} \cup M^{\prime}$ inside subalgebra $S^{\prime}=j \wedge S \wedge j$ by letting $J^{\prime}=\{j\}, M^{\prime}=j \wedge I \wedge j$ and setting $A^{\prime}\left[B^{\prime}\right]$ equal to the image of $M^{\prime}$ in $A \cap S^{\prime}$ [in $B \cap S^{\prime}$ ] under a single coset bijection of $S^{\prime}$ from $M^{\prime}$ to the intermediate class. While all pairs of elements from $A^{\prime}$ and $B^{\prime}$ join commute, not all pairs meet commute. 
3.6. If $C$ and $D$ are comparable equivalence classes in $S$, then by the coset cardinality of $C$ and $D$, denoted $c[C, D]$, is meant the common cardinality of all the cosets of $C \cup D$. Returning to the previous situation of classes $A, B, J$, and $M$, it is clear that $J$ is finite if both $c[J, A]$ and $c[J, B]$ are finite, in which case $|J|$ divides $c[J, A] c[J, B] / c[J, M]$, with equality holding when $S$ is symmetric. The dual assertion holds for $M$. It follows that the union of all finite classes of a skew lattice is a subalgebra; similarly, for a fixed prime $p$ the union of all finite classes having p-power order is a subalgebra. Skew lattices for which all classes have prime power order arise in finite dimensional algebras over finite fields. It is clear that a skew lattice is finite if its underlying lattice is finite and there exist finite classes $A_{1}, \ldots, A_{n}$ which form a set of generators on the lattice. By 2.4 , a finitely generated skew lattice need not be finite, even when its underlying lattice is finite. Orthogonality also implies that if $A$ and $B$ are finite classes with relatively prime orders, then $A$ and $B$ elementwise commute and generate a finite piecewise disconnected subalgebra, $A \cup B \cup J \cup M$, with $c[J, M]=1,|J|=c[J, A] c[J, B]$, and $|M|=c[M, A] c[M, B]$. In a finite dimensional algebra over a field, all cosets in an affine skew lattice have finite dimensions as hyperspaces, to be denoted $d[J, A], d[J, B]$, etc. One thus has $\operatorname{dim}(J)=d[J, A]+d[J, B]-d[J, M]$ and $\operatorname{dim}(M)=d[M, A]+d[M, B]-$ $d[J, M]$.

3.7. We significantly extend the context of our discussion. To begin, let $(S, \triangleright)$ be a quasi-ordered set. The quasi-order $\triangleright$ partitions $S$ into equivalence classes which are partially ordered as follows: $A \geq B$ iff $a \triangleright b$ for all $a$ in $A$ and $b$ in $B$. A partial skew lattice on $(S, \triangleright)$ is a quadruple $(S, \triangleright, \vee, \wedge)$ where $\vee$ and $\wedge$ are partial binary operations defined whenever both elements are comparable under the quasi-order, and such that the union of any chain of equivalence classes is a skew lattice under $\vee$ and $\wedge$, with equivalence class structure the same as that given by $(S, \triangleright)$. That $\vee$ and $\wedge$ are both associative on totally quasi-ordered subsets is referred to as linear associativity. One consequence of linear associativity is middle associativity: if $m \triangleleft x, y \triangleleft p$, then $(x \vee p) \vee y=x \vee(p \vee y)$, since both sides equal $(x \vee p) \vee(p \vee y)$, and likewise $(x \wedge m) \wedge y=x \wedge(m \wedge y)$.

3.8. Examples. (i) A degenerate partial skew lattice structure may be imposed on any quasi-ordered set by giving each equivalence class a rectangular structure compatible with the class cardinality and between strictly comparable elements $a \triangleright b$ setting $a \vee b=a=b \vee a$ and $a \wedge b=b=b \wedge a$. (ii) Let $A$ be a ring with idempotents $E(A)$. The right quasi-ordering of $E(A)$ is defined by $x \geq_{R} y$ iff $x y=y$. The standard partial skew lattice on $\left(E(A), \geq_{R}\right)$ is given by the standard operations: $x \vee y=x+y-x y$ and $x \wedge y=x y$. Dually one defines the left quasi-ordering of $E(A)$ by $x \geq_{L} y$ iff $y x=y$. Thus there are two standard partial skew lattice structures on the idempotents of $A$; however the complementation map, $x^{c}=1-x$, gives an affine dual isomorphism of the two partial algebras. (iii) Let $S$ be a skew lattice. The natural quasi-ordering of $S$ is defined by $x>_{N} y$ iff $x \vee y \vee x=x$, or equivalently, $y \wedge x \wedge y=y$. The canonical partial skew lattice of $S$ is $\left(S,>_{N}, \vee, \wedge\right)$, with the original operations restricted to elements comparable under the quasi-order. Theorem 3.3 implies that every skew lattice is determined by its canonical partial algebra.

3.9. When is a given partial skew lattice $(S, \triangleright, \vee, \wedge)$ canonical for some skew lattice? Two conditions are obviously necessary. The quasi-order has 
to be a quasi-lattice, that is, its equivalence classes must form a lattice under the induced ordering. In addition, each pair of equivalence classes must be orthogonal in their join and meet classes. Given these two conditions the partial operations on $S$ may be extended to full operations on $S$ with the join defined as $x \vee y=(x \vee q \vee x) \vee(y \vee p \vee y)$ for any $p \geq x$ and $q \geq y$, where $p$ and $q$ lie in the join class of $x$ and $y$, and the meet is defined as $x \wedge y=(x \wedge n \wedge x) \wedge(y \wedge m \wedge y)$ for any $m \leq x$ and $n \leq y$ in the meet class of $x$ and $y$. The full algebra $(S, \vee, \wedge)$ is called the algebraic closure of $(S, \triangleright, \vee, \wedge)$.

Theorem 3.10. A partial skew lattice is the canonical partial algebra of a skew lattice if and only if the quasi-order is a quasi-lattice and any pair of equivalence classes are orthogonal in both their join and meet classes, in which case the skew lattice is the algebraic closure.

Proof. Necessity is clear. For sufficiency we show the algebraic closure to be a skew lattice. So let $x, y$ be given. Then $x \vee y=x^{\prime} \vee y^{\prime}$ for some $x^{\prime}, y^{\prime}$ in the join class of $x$ and $y$, with $x \leq x^{\prime}$ and $y \leq y^{\prime}$. In the partial algebra, $x \wedge(x \vee y)=x \wedge\left(x^{\prime} \vee y^{\prime}\right)=x \wedge\left(x \vee x^{\prime} \vee y^{\prime}\right)=x$. The other cases of absorption are similarly verified. Suppose that $x, y \triangleleft s$. By middle associativity, $x \vee y=$ $x \vee q \vee p \vee y$ for all $p \geq x$ and $q \geq y$ in the join class of $x$ and $y$. Choose $q$ so that $y \leq q \leq y \vee s \vee y$. Then using linear associativity:

$$
\begin{aligned}
(x \vee y) \vee s & =(x \vee q \vee p \vee y) \vee s=(x \vee q) \vee p \vee(y \vee s \vee y) \vee s \\
& =(x \vee q) \vee(y \vee s \vee y) \vee s=x \vee(y \vee s) .
\end{aligned}
$$

We similarly obtain all other cases of outer associativity: if $r \triangleleft x, y \triangleleft s$, then also $s \vee(x \vee y)=(s \vee x) \vee y, r \wedge(x \wedge y)=(r \wedge x) \wedge y$, and $(x \wedge y) \wedge r=x \wedge(y \wedge r)$. With the available forms of conditional associativity, it is now easy to verify associativity. For instance, if $s$ lies in the join class of $x, y$, and $z$, then both $(x \vee y) \vee z$ and $x \vee(y \vee z)$ are seen to equal $x \vee[y \vee(z \vee s \vee x) \vee y] \vee z$. Thus the algebraic closure is a skew lattice and the theorem follows.

3.11. The major remaining question is properly posed as a question about partial skew lattices: when does a primitive covering of a quasi-ordered set $(S, \triangleright)$ determine a partial skew lattice? So suppose a rectangular skew lattice structure has been assigned to each equivalence class of $(S, \triangleright)$ and to each strictly comparable pair of classes $A>B$ a primitive skew lattice structure has been assigned which agrees with both rectangular structures and the class ordering. If $x \triangleright y$ in $S$ with $x$ in $A$ and $y$ in $B$, then define $x \vee y, y \vee x, x \wedge y$, and $y \wedge x$ to be as given in $A \cup B$. The resulting partial algebra, $(S, \triangleright, \vee, \wedge)$, obeys the idempotent laws and conditional absorption laws for comparable elements. The question thus becomes: under what conditions does linear associativity hold in $(S, \triangleright, \vee, \wedge)$ ? To answer this, consider the pair of classes $A>B$. If $B_{j}$ is a coset in $B$, then by the lower coset projection of $A$ onto $B_{j}$ is meant the map $p_{j}: A \rightarrow B$ projecting each element in $A$ to its unique image in $B_{j}$. Clearly this map has the form $p_{j}[a]=a \wedge b \wedge a$ for any $b$ in $B_{j}$. Likewise for each upper index $i$, there is an upper coset projection, $q_{i}$, of $B$ onto $A_{i}$ considered to be a function into $A$. If $A=B$, then set $p=q=1_{A}$. Let Proj $\downarrow(S)$ [respectively Proj $1(S)$ ] denote the family of all lower [upper] coset projections between comparable classes of $S$. If composites of successive pairs of lower [upper] coset projections are always coset projections, then $\operatorname{Proj} \mid(S)[\operatorname{Proj} 1(S)]$ forms the category of lower [upper] coset projections. 
Theorem 3.12. The partial algebra $(S, \triangleright, \vee, \wedge)$ induced from a primitive covering of a quasi-ordered set $(S, \triangleright)$ is a partial skew lattice precisely when both the family of lower coset projections, Proj $\downarrow(S)$, and the family of upper coset projections, Proj $1(S)$, form categories under ordinary composition.

Proof. Suppose we are given a primitive covering of $(S, \triangleright)$ for which Proj $\lfloor(S)$ and Proj $1(S)$ form categories under ordinary composition of functions. Given comparable classes $A>B>C$ in $S$, pick $a$ in $A, b$ in $B, c$ in $C$ and set $d=b \wedge c \wedge b$ in $B \cup C$. Using only primitive operations and projections:

$$
a \wedge(b \wedge c)=a \wedge(d \wedge c)=(a \wedge d) \wedge c=p_{d}[a] \wedge d \wedge c=p_{d}[a] \wedge c
$$

and

$$
\begin{aligned}
(a \wedge b) \wedge c & =\left(p_{b}[a] \wedge b\right) \wedge c=p_{c}\left(p_{b}[a] \wedge b\right) \wedge c \\
& =p_{c} p_{b}[a] \wedge p_{c}[b] \wedge c=p_{c} p_{b}[a] \wedge c
\end{aligned}
$$

where $p_{b}: A \rightarrow B, p_{c}: B \rightarrow C$, and $p_{d}: A \rightarrow C$ denote the coset projections containing $b, c$, or $d$ in their image. By assumption, either $p_{d}=p_{c} p_{b}$ or their images are disjoint in $C$. Pick $a$ in $A$ such that $a>b$; then $p_{c} p_{b}[a]=p_{c}[b]=$ $d$. Thus the images of $p_{d}$ and $p_{c} p_{b}$ overlap so that $p_{d}=p_{c} p_{b}$ and $a \wedge(b \wedge c)=$ $(a \wedge b) \wedge c$ for all $a$ in $A$. That $a \wedge(c \wedge b)=(a \wedge c) \wedge b$ follows from the fact that both sides must equal $(a \wedge c) \wedge(c \wedge b)$. Moreover, $(c \wedge a) \wedge b=c \wedge(a \wedge b)$ since both sides easily reduce to $c \wedge b$. All other instances of associativity amongst $a, b$, and $c$ in both $\wedge$ and $\vee$ are checked in a manner similar to one of the cases already considered. Conversely, if $(S, \triangleright, \vee, \wedge)$ is a partial skew lattice, then conditional biregularity yields $(a \wedge b \wedge a) \wedge c \wedge(a \wedge b \wedge a)=a \wedge(b \wedge c \wedge b) \wedge a$ for $a \triangleright b \triangleright c$ and thus $p_{c} p_{b}=p_{b \wedge c \wedge b}$. Hence Proj $\downarrow(S)$ forms a category; in similar fashion so does Proj $1(S)$.

3.13. Remarks. (i) Theorem 3.12 generalizes the picture given in [20] of a normal band as a small category of homomorphisms between rectangular bands. Whereas in a normal band there is exactly one coset projection from a higher class to a lower one, in the general case of a partial skew lattice there are multiple projections, but all with disjoint image sets in the lower [or upper] class. If $S$ is a skew lattice, then one has $x \vee y=q[x] \vee q^{\prime}[x]$ and $x \wedge y=p[x] \wedge p^{\prime}[x]$, where $q, q^{\prime}$ are projections into the join class and $p, p^{\prime}$ are projections into the meet class, with the precise projections determined by orthogonality. (ii) The small category of homomorphisms between rectangular bands which forms a normal band is itself the image of a functor from a meet semilattice to the category of rectangular bands. While this does not extend to partial skew lattices in general, there is a class of exceptions. A skew lattice $S$ is normal if each principal subalgebra $x \wedge S \wedge x$ is a sublattice. In this case given any comparable pair of classes, the lower class forms a unique coset and there is a unique lower coset projection between the two classees. As a consequence, one can obtain a close analogue of the Yamada-Kimura Theorem which pictures the entire skew lattice as created from a lattice domained functor. (See [12, Theorem 1.6]. The skew Boolean algebras studied in [11] are necessarily normal.) (iii) The interrelation between quasi-orders and [partial] skew lattice structures which they may support is a recurring theme in noncommutative lattice theory. (See [6,9, and 18].) This type of interrelation has played an important role in semigroup theory. Early in the development of topological semigroups, naturally totally quasi-ordered compact semigroups were studied by a number of authors. (For historical remarks 
see [7].) Natural quasi-orderings of the set of idempotents $E(S)$ of a regular $S$ have received considerable attention, especially since Nambooripad in [14] gave an abstract characterization of $E(S)$ in terms of the natural quasi-orders which is independent of any reference to the ambient semigroup. (His approach has some similarities to Schein's approach to pseudolattices in [18].) Partial bands defined on partially ordered sets appeared in Warne's work on bands in [19]. While each quasi-ordered set supports a partial skew lattice structure, the author knows no general answer to the question: which quasi-ordered sets support a skew lattice structure? A partial answer is implicit in 4.7 below.

3.14. Do coset bijections naturally form a category under the composition of partial bijections? Not always. Let the right-handed skew lattice $S$ be the union of three equivalence classes $R_{1}>R_{2}>R_{3}$, each a copy of the real numbers. Let the cosets for $R_{1}>R_{2}$ have the form [m,m+1), the cosets for $R_{2}>R_{3}$ have the form $[n-1 / 2, n+1 / 2)$, and the cosets for $R_{1}>R_{3}$ have the form [ $m, m+1)$ in $R_{1}$ and $[n-1 / 2, n+1 / 2)$ in $R_{3}$. In all cases the coset bijection takes an element to the unique element sharing the same decimal part. Thus 4.2 goes to the number of the form $n+.2$. Upon composing coset bijections one obtains "half" bijections for $R_{1}>R_{3}$ as well as empty bijections. In general, nonempty composites of coset bijections refine coset bijections between the outer classes, and cosets which the outer classes induce on each other refine cosets of the middle class in the outer classes.

3.15. A partial skew lattice $(S, \triangleright, \vee, \wedge)$ is categorical if nonempty composites of coset bijections are coset bijections. In this case we define the category of coset bijections, denoted $\operatorname{CSB}(S, \triangleright)$, as follows: its class of objects is the set of equivalence classes of $S$ endowed with their rectangular structure and with the identity maps being their identity morphisms; for strictly comparable classes $A>B$ the morphism set consists of the coset bijections between $A$ and $B$ plus (in the case of empty composites) an $A-B$ labelled copy of the empty partial bijection; composition is ordinary composition of partial bijections with empty composites given the appropriate labelling to avoid confusing empty partial bijections in different morphism sets.

Theorem 3.16. The following hold

(i) A partial skew lattice is categorical iff for any given $x \equiv p \geq q \geq r$,

$$
\begin{aligned}
& (x \wedge r \wedge x) \vee q \vee(x \wedge r \wedge x) \\
& \quad=[(x \wedge r \wedge x) \vee p \vee(x \wedge r \wedge x)] \wedge q \wedge[(x \wedge r \wedge x) \vee p \vee(x \wedge r \wedge x)] .
\end{aligned}
$$

(ii) Categorical skew lattices form a subvariety of skew lattices.

(iii) In a ring, any partial skew lattice of idempotents using the standard join and meet operations is categorical.

(iv) In a ring $A$ the complementation map, $x^{c}=1-x$, induces a dual isomorphism of $\operatorname{CSB}\left(E(A), \geq_{R}\right)$ with $\operatorname{CSB}\left(E(A), \geq_{L}\right)$.

Proof. To see (i), observe that the chain $p \geq q \geq r$ is a typical piece of a nonempty composition of coset bijections, $x \wedge r \wedge x$ is a typical member of the coset of the outer classes at $r,(x \wedge r \wedge x) \vee p \vee(x \wedge r \wedge x)$ is the image of $x \wedge r \wedge x$ in the top coset containing $p$, and the conditional identity is what is needed to insure that the correspondence $(x \wedge r \wedge x) \vee p \vee(x \wedge r \wedge x) \rightarrow x \wedge r \wedge x$ is the result of a composite bijection involving middle cosets containing $q$. 
In a skew lattice the conditional identity is easily replaced by a rather lengthy unconditional identity and (ii) follows. (iii) follows from (i) and (iv) is trivial.

\section{FURTHER EXAMPLES AND RESULTS}

4.1. A skew lattice with totally ordered equivalence classes is called a skew chain. A skew chain with $n+1$ equivalence classes is said to have length $n$. Skew chains of finite length directly generalize primitive skew lattices with the latter being the skew chains of length one. We examine the structure of skew chains arising in rings. To begin, let $e_{n}>\cdots>e_{0}$ be a chain of idempotents of length $n$ in ring $A$. The right extension of $e_{n}>\cdots>e_{0}$, to be denoted $P\left[e_{n}, \ldots, e_{0}\right]$, is the skew chain which is the union of the $R$-sets $R\left(e_{i}\right)$. For the primitive case, $n=1$, recall that the structure of $P[e, f]$ is encoded in a bilinear map $\theta: B \times A \rightarrow C$ which yields a coordinatization, $P[A, B, C, \theta]$, of $P[e, f]$. In the general case, the structure of $P\left[e_{n}, \ldots, e_{0}\right]$ is given by the following $n$-fold generalization of what occurs in the primitive case.

4.2. Construction. A bilinear complex over commutative ring $K$ is a doubly indexed family of $K$-modules $\mathbf{D}=\left\{D_{i j} \mid 0 \leq i<j \leq n+1\right\}$ connected by a triply indexed family of $K$-bilinear maps $\mathbf{u}=\left\{u_{i j k}: D_{i j} \times D_{j k} \rightarrow D_{i k} \mid 0 \leq i<\right.$ $j<k \leq n+1\}$. Elements of $D_{i j}$ are denoted by $a_{i j}, b_{i j}, \ldots$ and evaluations $u_{i j k}\left(a_{i j}, b_{j k}\right)$ are denoted by $a_{i j} b_{j k} .(\mathbf{D}, \mathbf{u})$ is coherent if for all $i<j<k<l$ associativity holds: $\left(a_{i j} b_{j k}\right) c_{k l}=a_{i j}\left(b_{j k} c_{k l}\right)$. In this case set $S=\cup R_{p}$, where $R_{p}=\bigoplus\left\{D_{i j} \mid i \leq p<j\right\}$ for $0 \leq p \leq n$. Each subunion $R_{p} \cup R_{q}$ where $p<q$ may be given a right primitive structure with $R_{p}<R_{q}$ as follows. First set $C_{p q}=\bigoplus\left\{D_{i j} \mid i \leq p<q<j\right\}, A_{p q}=\bigoplus\left\{D_{i j} \mid p<i \leq q<j\right\}$, and $B_{p q}=\bigoplus\left\{D_{i j} \mid i \leq p<j \leq q\right\}$. Clearly $R_{p}=C_{p q} \oplus B_{p q}$ and $R_{q}=C_{p q} \oplus A_{p q}$. Next let $u^{p q}: B_{p q} \times A_{p q} \rightarrow C_{p q}$ be the matrix product: $u^{p q}\left(\sum b_{i j}, \sum a_{k l}\right)=$ $\sum_{j=k} b_{i j} a_{k l}$ where the $b_{i j} a_{k l}$ with common outer indices are added in their module summand. $u^{p q}$ is a $K$-bilinear map which imposes a right primitive skew lattice structure on $R_{p} \cup R_{q}$. The various primitive subalgebras together form a primitive covering of $S$ and since $(\mathbf{D}, \mathbf{u})$ is coherent, this covering yields a skew chain of length $n$, to be denoted $S[\mathbf{D}, \mathbf{u}]$. This construction is called the $n$-fold bilinear construction. Observe that for $n=0$ one obtains a rectangular algebra on $D_{01}$ and for $n=1$ one obtains the right primitive algebra $P\left[D_{12}, D_{01}, D_{02}, u_{012}\right]$. Thus this construction indeed generalizes the standard coordinatization procedure for affine right primitive skew lattices in rings; however the full justification of this construction depends upon the following considerations.

4.3. Let $A$ be a ring for which $K$ is a subring of the center and let $e_{n}>\cdots>$ $e_{0}$ be a chain of idempotents in $E(A)$. Setting $e_{n+1}=1 \geq e_{n}$ and $e_{-1}=0 \leq e_{0}$, we form a coherent bilinear complex as follows: let $D_{i j}=\left(e_{i}-e_{i-1}\right) A\left(e_{j}-e_{j-i}\right)$ for $0 \leq i<j \leq n+1$, and let each $u_{i j k}$ be given by ring multiplication for $i<j<k$. This complex is called the canonical bilinear complex of the chain $e_{n}>\cdots>e_{0}$.

Theorem 4.4. Let $A$ be a ring for which $K$ is a subring of the center and let $(\mathbf{D}, \mathbf{u})$ be the canonical bilinear complex induced from the chain of idempotents $e_{n}>\cdots>e_{0}$. Then the skew chain $S[\mathbf{D}, \mathbf{u}]$ is isomorphic with the right extension $P\left[e_{n}, \ldots, e_{0}\right]$. Moreover, to within isomorphism every coherent bilinear complex is obtained canonically from a finite chain of idempotents in a ring. 
Proof. The isomorphism is defined on classes $R_{p} \rightarrow R\left(e_{p}\right)$ by $\sum a_{i j} \rightarrow e_{p}+$ $\sum a_{i j}$, where the first sum is direct summation in $R_{p}$ and the second sum is ring addition. Given any coherent complex $(\mathbf{D}, \mathbf{u})$, extend $\mathbf{D}$ to a square indexed array of $K$-modules by setting each $D_{i i}=K$ and setting $D_{i j}=0$ when $i>j$. Accordingly extend $\mathbf{u}$ by letting the $u_{i i j}$ and $u_{i j j}$ be scalar multiplication and letting the remaining new $u_{i j k}$ be zero maps. Associativity must hold in the extended complex. Let $A$ be the ring of formal $(n+2) \times(n+2)$ matrices $\left[a_{i j}\right]$, with $a_{i j}$ in $D_{i j}$ for $0 \leq i, j \leq n+1$. The matrices have the obvious addition; using the $u_{i j k}$ they may be multiplied in the usual matrix fashion. Set $e_{p}=\left[d(p)_{i j}\right]$, for $0 \leq p \leq n$, where $d(p)_{i j}=1$ if $i=j \leq p$ but 0 otherwise. The canonical complex induced from $e_{n}>\cdots>e_{0}$ is a copy of the original $(\mathbf{D}, \mathbf{u})$.

4.5. Thus we may, in effect, answer questions about skew chains in rings by analyzing coherent bilinear complexes. We do so as follows:

(i) The n-fold bilinear construction produces a piecewise disconnected skew chain $S[\mathbf{D}, \mathbf{u}]$ iff each $u_{i j k}=0$. On the other hand, a skew chain $S[\mathbf{D}, \mathbf{u}]$ is piecewise connected iff $[\mathbf{D}, \mathbf{u}]$ is generated by its superdiagonal modules, i.e., each $D_{i j}$ is generated by the $\mathbf{u}$-product $D_{i, i+1} \ldots D_{j-1, j}$.

(ii) Given a superdiagonal indexing of $K$-modules $F_{01}, \ldots, F_{n, n+1}$ a coherent bilinear complex $(\mathbf{F}, \mathbf{u})$ is freely constructed from them by first setting $F_{i j}=F_{i, i+1} \otimes \cdots \otimes F_{j-1, j}$, where $\otimes$ denotes the tensor product, and then letting $u_{i j k}: F_{i j} \times F_{j k} \rightarrow F_{i k}$ be the tensor map. The induced skew chain $S[\mathbf{F}, \mathbf{u}]$ is piecewise connected. An example of the free construction occurs when each $F_{i j}$ is $K$ for $i<j$ and each $u_{i j k}$ is just multiplication for $i<j<k$. In this case $(\mathbf{F}, \mathbf{u})$ is a copy of the canonical bilinear complex induced from $e_{n}>\cdots>e_{0}$, where $1>e_{n}>\cdots>e_{0}>0$ is any maximal chain of subidentity matrices in $K^{(n+2) \times(n+2)}$.

(iii) Let $C_{0, n+1}$ be the submodule of $D_{0, n+1}$ generated by the various uproducts $D_{0, i} D_{i, n+1}$ where $0<i<n+1$. Then upon setting $C_{i j}=D_{i j}$ in all other cases, the subcomplex $\left(\mathbf{C}, \mathbf{u}^{\prime}\right)$ induces the component algebra $S\left[\mathbf{C}, \mathbf{u}^{\prime}\right]$ of $S[\mathbf{D}, \mathbf{u}]$. Because $S[\mathbf{D}, \mathbf{u}]$ is homogeneous, all its components are isomorphic copies of $S\left[\mathbf{C}, \mathbf{u}^{\prime}\right]$.

4.6. One can easily generalize the concept of a skew chain and thus obtain a class of algebras which includes piecewise connected skew lattices. Recall that the center of a skew lattice $S$ coincides with the union of all singleton equivalence classes; an equivalence class is reducible when it is either the join or meet class of incomparable classes. A generalized skew chain is a skew lattice whose reducible classes lie in its center and thus its nontrivial classes lie in $\operatorname{Irr}(S)$, the union of the irreducible classes of $S$. Trivially, all skew chains are generalized skew chains. Due to orthogonality, all piecewise connected skew lattices are generalized skew chains. Generalized skew chains are always freely determined by their irreducible part. To understand the latter, consider a typical $\operatorname{Irr}(S)$. Two classes $A>B$ in $\operatorname{Irr}(S)$ are said to be separated if there is a reducible class of $S$ between them, in which case $A \cup B$ must be a degenerate subalgebra. Separation between $A$ and $B$ will occur when there is a third class in $S$ comparable to $A$ or $B$, but not to both. Thus $\operatorname{Irr}(S)$ is partitioned into basic skew chains: skew chains which are maximal subject to having pairwise inseparable classes; moreover, $\operatorname{Irr}(S)$ is freely determined by its basic skew chains. 
Theorem 4.7. Let $T$ be a lattice. To each basic chain in $\operatorname{Irr}(T)$ assign an overlying skew chain. Let $\operatorname{Irr}(S)$ be the union of these overlying skew chains and extend the skew chain operations so that comparable pairs of classes in $\operatorname{Irr}(S)$ lying in distinct skew chains form degenerate subalgebras. Then $\operatorname{Irr}(S)$ is a partial skew lattice whose partial operations can be extended uniquely to give a generalized skew chain structure on $S=\operatorname{Irr}(S) \cup(T-\operatorname{Irr}(T))$, having as irreducible part, $\operatorname{Irr}(S)$, and having as basic skew chains the assigned over-lying skew chains. $S$ is piecewise connected precisely when the assigned skew chains are piecewise connected. All generalized skew chains [piecewise connected skew lattices] may be obtained in this manner.

4.8. While piecewise disconnected skew lattices have rather trivial primitive subalgebras, their global structure need not be trivial. Moreover, piecewise disconnected skew lattices form a subvariety of skew lattices. Indeed, these skew lattices may be characterized by the implication: if $x \equiv x^{\prime}, y \equiv y^{\prime}$, and $x \geq y$, then $y \vee x^{\prime} \vee y \geq x \wedge y^{\prime} \wedge x$; this implication is then easily turned into a pair of lengthy identities. Normal skew lattices are piecewise disconnected as is any symmetric skew lattice on two generators.

4.9. The growing list of varieties of skew lattices raises the question: what can be said about the lattice of varieties, or at least about suitable chunks of this lattice? In the case of bands this question has been answered by several authors. (See [1 and 3-5].) Petrich in [14] classified varieties of bands determined by identities in at most three variables; the corresponding classification for skew lattices could be a formidable task. A related problem is that of describing finitely generated free skew lattices in a given variety. It is known that finitely generated bands are finite, whereas the free lattice on three generators is infinite. It turns out that the free [symmetric] skew lattice on two generators is infinite. We conclude this paper with a classification of all symmetric skew lattices on two generators. To this end let 2 denote the lattice $1^{0}$, let $\mathbf{R}$ denote the right rectangular skew lattice on $\{a, b\}$ and let $\mathbf{L}=\mathbf{R}^{*}$ denote its left-handed dual.

Theorem 4.10. The categorical symmetric skew lattices with two generators are copies of one of the following: $\mathbf{R}, \mathbf{L}, \mathbf{R} \times \mathbf{L}, \mathbf{2}, \mathbf{R} \times \mathbf{2}, \mathbf{L} \times \mathbf{2}, \mathbf{R} \times \mathbf{L} \times \mathbf{2}, \mathbf{R} \times \mathbf{2} \times$ $\mathbf{2}, \mathbf{L} \times \mathbf{2} \times \mathbf{2}$, and $\mathbf{R} \times \mathbf{L} \times \mathbf{2} \times \mathbf{2}$, the free categorical symmetric skew lattice on two generators.

4.11. Note that all skew lattices listed in the theorem are binormal. To classify the noncategorical cases we begin by letting $\mathbf{S}_{m, n}$, for $1 \leq m, n \leq \infty$, be the right handed skew lattice with join class $J=\left\{0^{+}, 1^{+}\right\}$, meet class $M=$ $\left\{0^{-}, 1^{-}\right\}$, and incomparable classes $A$ and $B$ where: $A=\mathbf{Z}_{2 m}$ if $m$ is finite, $A=\mathbf{Z}$ if $m$ is infinite; likewise, $B=\mathbf{Z}_{2 n}$ if $n$ is finite and $B=\mathbf{Z}$ otherwise. All elements of $B$ are denoted in prime form (e.g. $6^{\prime}$ ) to distinguish them from elements in $A$. The natural ordering of $\mathbf{S}_{m, n}$ is given by $0^{+}>2 i,(2 j)^{\prime}>0^{-}$ and $1^{+}>2 i+1,(2 j+1)^{\prime}>1^{-}$. $J$-cosets in $A$ and $B$ have the form $\{2 i-1,2 i\}$ and $M$-cosets in both classes have the form $\{2 i, 2 i+1\} . \mathbf{S}_{m, n}$ is the right-handed skew lattice determined by this coset geometry. Observe that $\mathbf{S}_{m, n}$ is generated by pairs of elements of the form $\left\{2 i,(2 j+1)^{\prime}\right\}$ or the form $\left\{(2 i)^{\prime}, 2 j+1\right\}$. Observe also that if one switches the $J$ and $M$ coset decompositions of either $A$ or $B$, then the new skew lattice is isomorphic with the old. Finally observe that $\mathbf{S}_{1,1}$ is just a copy of $\mathbf{R} \times \mathbf{2} \times \mathbf{2}$. Upon letting 
$\mathbf{T}_{m, n}$ denote the left handed dual of $\mathbf{S}_{m, n}$ and letting $\mathbf{S}[m, n ; p, q]$ denote the fibered product $\mathbf{S}_{m, n} x_{\mathbf{2} \times \mathbf{2}} \mathbf{T}_{p, q}$ we have

Theorem 4.12. Every noncategorical symmetric skew lattices on two generators is a copy of one of the following. $\mathbf{S}_{m, n}, \mathbf{L} \times \mathbf{S}_{m, n}, \mathbf{T}_{p, q}, \mathbf{R} \times \mathbf{T}_{p, q}$, or $\mathbf{S}[m, n ; p, q]$ where $3 \leq m+n, p+q \leq \infty$. $\mathbf{S}[\infty, \infty ; \infty, \infty]$ is the free symmetric skew lattice on two generators; it has four components, none of which are finitely generated; its components are all isomorphic, but $\mathbf{S}[\infty, \infty ; \infty, \infty]$ is not homogeneous.

Proof of 4.10, 4.12. Let $S$ be symmetric with generators $a$ and $b$ lying in classes $A$ and $B$ respectively. We need only treat the case in which $S$ is right handed (see $[10,1.14-1.16])$ and $A$ is incomparable with $B$. In general, right handed symmetry is equivalent to the identities $(x \wedge y) \vee x \vee y=y \vee x$ and $x \wedge y \wedge(x \vee y)=y \wedge x$. It follows that $J=\{a \vee b, b \vee a\}$ and $M=\{a \wedge b, b \wedge a\}$, with the coset bijection given by: $a \vee b \rightarrow b \wedge a$ and $b \vee a \rightarrow a \wedge b$. Moreover, $A$ is generated by $\{a\} \cup J \cup M$ while $B$ is generated by $\{b\} \cup J \cup M$. Thus if $a$ and $b$ commute one has a copy of $2 \times 2$; otherwise $S$ is forced to have the form $\mathbf{S}_{m, n}$, which in the case $m=n=1$ is a copy of $\mathbf{R} \times 2 \times \mathbf{2}$. Clearly $\mathbf{S}_{\infty, \infty}$ is the free right-handed symmetric skew lattice on two generators. Since both of its components are isomorphic, but need infinitely many generators, $\mathbf{S}_{\infty, \infty}$ cannot be homogeneous.

Corollary 4.13. In a symmetric skew lattice every pair of elements generates a piecewise disconnected subalgebra. If the skew lattice is also categorical, then the subalgebra is binormal. (Categorical symmetric skew lattices having three generators need not be binormal; by Theorem 2.4, those with four generators need not be piecewise disconnected.)

\section{REFERENCES}

1. A. P. Birjukov, Varieties of idempotent semigroups, Algebra i Logika 9 (1970), 255-273. (Russian)

2. A. H. Clifford, and G. B. Preston, The algebraic theory of semigroups, Vol. I, Math. Surveys, vol 7, Amer. Math. Soc. Providence, R.I., 1961.

3. C. F. Fennemore, All varieties of bands. I, Math. Nachr. 48 (1971), 237-252.

4. __ All varieties of bands. II, Math. Nachr. 48 (1971), 253-363.

5. J. A. Gerhard, The lattice of equational classes of idempotent semigroups, J. Algebra $\mathbf{1 5}$ (1970), 195-224.

6. M. D. Gerhardts, Schragverbande und Quasiordnungen, Math. Ann. 181 (1969), 65-73.

7. K. H. Hofmann and P. S. Mostert, Elements of compact semigroups, Merrill, Washington, D.C., 1966.

8. J. M. Howie, An introduction to semigroup theory, Academic Press, 1976.

9. P. Jordan, The mathematical theory of quasi-order, Semigroups of Idempotents and Noncommutative Lattices-A New Field of Algebra, 1961 Report, European Office of Aerospace Research.

10. J. Leech, Skew lattices in rings, Algebra Universalis 26 (1989), 48-72.

11. __ Skew Boolean algebras, Algebra Universalis 27 (1990), 497-506.

12. __ Normal skew lattices, Semigroup Forum 44 (1992), 1-8.

13. E. S. Ljapin, Semigroups, Transl. Math. Monographs, vol. 3, Amer. Math. Soc., Providence, R.I., 1963.

14. K. S. S. Nambooripad, Structure of regular semigroups, Mem. Amer. Math. Soc. 224 (1979).

15. M. Petrich, A construction and classification of bands, Math. Nachr. 48 (1971), 263-274. 
16. _ _ Introduction to semigroups, Bell and Howell, 1973.

17. _ Lectures on semigroups, Wiley, 1977.

18. B. M. Schein, Pseudosemilattices and pseudolattices, Amer. Math. Soc. Transl. (2) 119 (1983), 1-16.

19. R. J. Warne, On the structure of idempotent semigroups, Proc. Amer. Math. Soc. 37 (1973), 17-21.

20. M. Yamada and N. Kimura, Note on idempotent semigroups. II, Proc. Japan Acad. 34 (1958), 110-112.

Department of Mathematics, Westmont College, Santa Barbara, California 93108

E-mail address: leech@westmont.edu 